\section{Clinical Trials In Progress}

\section{A GLOBAL, MOLECULAR DISEASE CHARACTERIZATION INITIATIVE (MDCI) IN ONCOLOGY CLINICAL TRIALS}

${ }^{1}$ Melissa Johnson*, ${ }^{2}$ Adrian Sacher, ${ }^{2}$ Marcus Butler, ${ }^{3}$ Hassane Zarour, ${ }^{4}$ Jeffrey Weber ${ }^{5}$ Edward Garon, ${ }^{6}$ David Carbone, ${ }^{7}$ Arindam Dhar, ${ }^{7}$ Cristina Messina, ${ }^{7}$ Roma Patel, ${ }^{7}$ Kristin Blouch, ${ }^{8}$ Axel Hoos, ${ }^{7}$ Anne-Marie Martin. ${ }^{1}$ Sarah Cannon Research Institute LLC, Nashville, TN, USA; ${ }^{2}$ Princess Margaret Cancer Centre, Toronto, Canada; ${ }^{3}$ University of Pittsburgh Medical Center, Pittsburgh, PA, USA; ${ }^{4}$ NYU Grossman School of Medicine, New York, NY, USA; ${ }^{5}$ David Geffen School of Medicine, University of California, Los Angeles, Santa Monica, USA; ${ }^{6}$ The Ohio State University, Columbus, OH, USA; ${ }^{7}$ GlaxoSmithKline, Collegeville, PA, USA; ${ }^{8}$ GlaxoSmithKline (at the time of publication), Collegeville, PA, USA

Background To address the heterogeneity of response to therapies, the oncology field is moving toward precision medicine (PM), with therapy tailored to a patient's disease. Biomarker assessment is now critical when screening for enrollment into clinical studies, such as for T-cell receptor therapies and combination studies, and determining status of a single analyte does not always correlate to clinical benefit. ${ }^{1}$ This shift may increase enrollment screen-failure rates due to restrictive eligibility criteria (e.g., biomarker prevalence). For oncology patients, the need for timely treatment does not allow for a sequential, complex, and time-consuming screening process for each individual clinical trial. The design and data from studies such as Lung MAP have emphasized a need to change clinical trial screening. As we begin to target less common genomic and immunotherapy subtypes, comprehensive molecular characterization may lead to rapid delivery of therapies to patients while maximizing the quality of data. ${ }^{2}$ The Molecular Disease Characterization Initiative (MDCI; GlaxoSmithKline Study 213299 [NCT04772053]) screens for multiple studies at once by collecting a baseline assessment of disease prior to treatment with different therapeutic modalities, and evaluating the patient's tumor and blood genetics. The MDCI creates a platform to accelerate the availability of new therapeutic options for patients through matched investigative and PM clinical trials, while building a scientific database to facilitate the investigation of biological mechanisms underpinning clinical outcomes.

Methods This multicenter study enrolls patients $(\mathrm{N}=400)$ with advanced/metastatic malignancies, initially non-small cell lung cancer, to collect biospecimens for broad molecular profiling and examining the expression of specific antigens (e. g. NY-ESO-1, LAGE-1a), immune markers (e.g. ICOS, PD-L1), tumor-infiltrating immune cells, differentially expressed genes, and human leukocyte antigen (HLA). In this single-arm, noninterventional, molecular analysis study there is no administration of investigational product. MDCI interrogates patient tumor specimens using CLIA-validated immunohistochemistry assays coupled with next-generation sequencing of DNA and RNA. Blood is also collected for assessment (e.g. HLA typing, ctDNA). Based on this molecular profiling and previous medical history, potential clinical trial options are returned to both physicians and patients with the aim to reduce time-to-treatment (figure 1). Participants may be subsequently matched to ongoing clinical trials of novel immunotherapeutics or adoptive cell therapy. Patients who do not match to a clinical trial but continue with standard of care may choose longitudinal follow-up and re-analyses of disease at progression.

Acknowledgements Study 213299 (NCT04772053) is funded by GlaxoSmithKline.

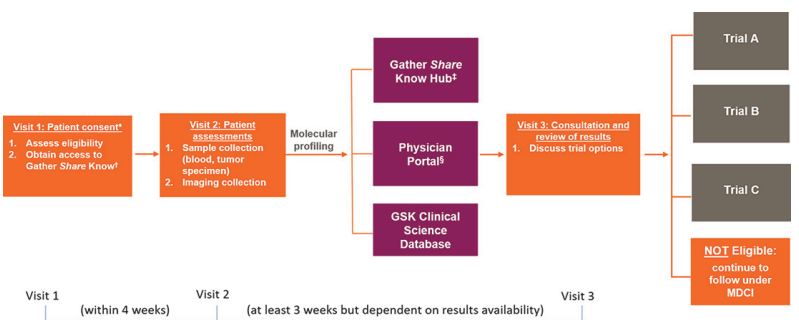

Abstract 390 Figure $1 \mathrm{MDCl}$ study design schema. ${ }^{*} \mathrm{MDCl}$ will be an optional protocol under select GSK oncology protocols. †Gather Share Know is an optional patient-facing portal where patients can view subsets of their results. $\$$ Subset of data to be shared on Gather Share Know. §Patient's molecular profile and medical history will be compared against the eligibility criteria of select GSK oncology protocols to identify potential trial options. $\mathrm{MDCl}$, Molecular Disease Characterization Initiative.

\section{REFERENCES}

1.. Blons H, Garinet S, Laurent-Puig P, Oudart JB. Molecular markers and prediction of response to immunotherapy in non-small cell lung cancer, an update. J Thorac Dis 2019;11(Suppl 1):S25-S36.

2.. Lam, Vincent K, Papadimitrakopoulou V. Master protocols in lung cancer: experience from lung master protocol. Curr Opin Oncol 2018;30:p92-97.

Ethics Approval The study was approved by Advarra Central IRB, approval date 4 January 2021.

http://dx.doi.org/10.1136/jitc-2021-SITC2021.390 